\title{
Efficient heating of single-molecule junctions for thermoelectric studies at cryogenic temperatures
}

Cite as: Appl. Phys. Lett. 115, 073103 (2019); https://doi.org/10.1063/1.5118861

Submitted: 05 July 2019 . Accepted: 27 July 2019 . Published Online: 13 August 2019

Pascal Gehring, Martijn van der Star, Charalambos Evangeli (D), Jennifer J. Le Roy, Lapo Bogani, Oleg V. Kolosov (D), and Herre S. J. van der Zant (iD)
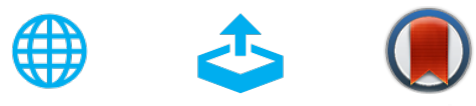

View Online

Export Citation

\section{ARTICLES YOU MAY BE INTERESTED IN}

Solvent-free synthesis of organometallic halides $\mathrm{CH}_{3} \mathrm{NH}_{3} \mathrm{Pbl}_{3}$ and $\left(\mathrm{CH}_{3} \mathrm{NH}_{3}\right)_{3} \mathrm{Bi}_{2} \mathrm{l} 9$ and their thermoelectric transport properties

Applied Physics Letters 115, 072104 (2019); https://doi.org/10.1063/1.5113535

Manifestation of the electromagnetic proximity effect in superconductor-ferromagnet thin film structures

Applied Physics Letters 115, 072602 (2019); https://doi.org/10.1063/1.5114689

Electrically tuneable exciton energy exchange between spatially separated 2-dimensional semiconductors in a microcavity

Applied Physics Letters 115, 071103 (2019); https://doi.org/10.1063/1.5110492

\section{Applied Physics Letters}

Mid-IR and $\mathrm{THz}$ frequency combs special collection 


\title{
Efficient heating of single-molecule junctions for thermoelectric studies at cryogenic temperatures
}

\author{
Cite as: Appl. Phys. Lett. 115, 073103 (2019); doi: 10.1063/1.5118861 \\ Submitted: 5 July 2019 . Accepted: 27 July 2019 . \\ Published Online: 13 August 2019
}
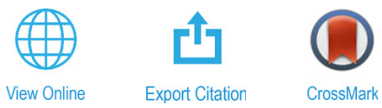

Pascal Gehring, ${ }^{1, a)}$ Martijn van der Star, ${ }^{7}$ Charalambos Evangeli, ${ }^{2,3}$ (D) Jennifer J. Le Roy, ${ }^{2}$ Lapo Bogani, ${ }^{2}$ Oleg V. Kolosov, ${ }^{3}$ (D) and Herre S. J. van der Zant ${ }^{7}$

\author{
AFFILIATIONS \\ ${ }^{7}$ Kavli Institute of Nanoscience, Delft University of Technology, Lorentzweg 1, 2628 CJ Delft, The Netherlands \\ ${ }^{2}$ Department of Materials, University of Oxford, Parks Road, OX1 3PH Oxford, United Kingdom \\ ${ }^{3}$ Department of Physics, Lancaster University, Bailrigg, LA1 4YB Lancaster, United Kingdom

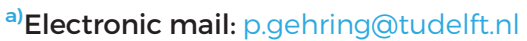

\begin{abstract}
The energy dependent thermoelectric response of a single molecule contains valuable information about its transmission function and its excited states. However, measuring it requires devices that can efficiently heat up one side of the molecule while being able to tune its electrochemical potential over a wide energy range. Furthermore, to increase junction stability, devices need to operate at cryogenic temperatures. In this work, we report on a device architecture to study the thermoelectric properties and the conductance of single molecules simultaneously over a wide energy range. We employ a sample heater in direct contact with the metallic electrodes contacting the single molecule which allows us to apply temperature biases up to $\Delta T=60 \mathrm{~K}$ with minimal heating of the molecular junction. This makes these devices compatible with base temperatures $T_{\text {bath }}<2 \mathrm{~K}$ and enables studies in the linear $\left(\Delta T \ll T_{\text {molecule }}\right)$ and nonlinear $\left(\Delta T \gg T_{\text {molecule }}\right)$ thermoelectric transport regimes.
\end{abstract}

Published under license by AIP Publishing. https://doi.org/10.1063/1.5118861

Theory predicts that electrical and thermoelectric properties of single molecules can be tailored by chemical design. For example, adding pendant groups to a conjugated molecule backbone can introduce sharp features in its energy dependent transmission probability, because of quantum interference effects, ${ }^{1}$ and such sharp features should generate exceptionally high thermoelectric efficiencies. ${ }^{2}$ Furthermore, single molecules can host a rich variety of physical effects: ${ }^{3}$ strong electron-phonon interactions, ${ }^{4}$ strong correlations and Kondo effects, ${ }^{5}$ or exotic blockade phenomena. ${ }^{6}$ All these are predicted to strongly influence the thermoelectric properties, ${ }^{7-10}$ but these predictions remain untested, because of a lack of appropriate experimental platforms.

In order to perform detailed thermoelectric characterizations of single molecules, the device architecture needs to fulfill the following conditions: the device needs to be compatible with methods to contact single molecules; a gate electrode is necessary for a full characterization of the thermoelectric properties of the single-molecule junction; because of the thermal instabilities in molecular junctions, the devices need to be compatible with cryogenic temperatures; and for the same reason, the temperature difference between the hot and the cold side $\Delta T=T_{\text {hot }}-T_{\text {cold }}$ in the molecular junction must not heat excessively the molecule itself. So far, only a few device architectures exist that fulfill some of the aforementioned conditions, based on graphene ${ }^{11}$ or Au electrodes. ${ }^{12}$ These devices suffer, however, from low heating efficiencies $\left(50-150 \mathrm{mK} \mathrm{mW}^{-1}\right)$ and, in devices with a side heater, the temperature profile along the channel is approximately linear ${ }^{13}$ so that high heater powers are necessary to apply $\Delta T$ across short junctions. For the case of graphene junctions, a side heater produces strong heating of the cold side of the junction, characterized by $\left(T_{\text {cold }}-T_{\text {bath }}\right) /$ $\Delta T \approx 5$, where $T_{\text {bath }}$ is the temperature of the cryostat. This makes these devices not compatible with measurements at low cryogenic temperatures.

Here, we develop a device architecture for simultaneously studying the electric and thermoelectric properties of single molecules as a function of the gate voltage $V_{g}$. Fabrication is based on electromigration and self-breaking of $\mathrm{Au}$, leading to several key advantages: $\mathrm{Au}$ enables access to different tunnel coupling strengths (e.g., by using thiol bonds, and spacer linkers); ${ }^{14}$ self-breaking ${ }^{15}$ of the Au bridges can prevent the formation of spurious quantum dots, which is sometimes a problem for carbon-based leads; ${ }^{16}$ the close proximity of the sample heater to the leads enables efficient heat transfer, while reducing heating of the single molecule at temperature $T_{\text {molecule }}=\left(T_{\text {hot }}+T_{\text {cold }}\right) / 2$ (thus 
ensuring device stability) and enabling experiments at $T_{\text {bath }}<2 \mathrm{~K}$. The improved heating efficiency also provides access to a wide $\Delta T$ range (millikelvin to few tens of kelvin), opening the way to the study of the thermoelectric properties of single-molecule junctions in the linear $\left(\Delta T \ll T_{\text {molecule }}\right)$ and nonlinear $\left(\Delta T \gg T_{\text {molecule }}\right)$ thermal bias regimes. Moreover, this method allows the simultaneous measurement of the gate-dependent conductance $G\left(V_{\mathrm{g}}\right)$ and thermoelectric current $I_{\mathrm{th}}\left(V_{\mathrm{g}}\right)$. This eliminates the problem that small drifts of the signals (because of hysteresis effects of the gates or activation of charge traps in the gate oxide) can hinder a direct comparison of data sets when the two quantities are measured subsequently, as in previous devices.

The junctions are fabricated following the scheme depicted in Figs. 1(a)-1(d). A Pd sample heater ( $3 \mathrm{~nm} \mathrm{Ti} / 27 \mathrm{~nm} \mathrm{Pd})$ and a Pd gate electrode $(1 \mathrm{~nm} \mathrm{Ti} / 6 \mathrm{~nm} \mathrm{Pd})$ were patterned on a Si wafer with $817 \mathrm{~nm}$ $\mathrm{SiO}_{2}$ using standard electron beam lithography and electron beam evaporation [Fig. 1(a)]. A thin gate electrode is used to reduce thermal transport between drain and source lead. Pd is used because it is known to form uniform thin layers with low surface roughness. ${ }^{17}$ In a second step a $10 \mathrm{~nm} \mathrm{Al}_{2} \mathrm{O}_{3}$ insulating layer is globally applied by atomic layer deposition [Fig. 1(b)]. This layer serves as a gate dielectric and as an insulation layer to electrically insulate the sample heater from the drain and source leads. ${ }^{18}$ Thereafter, a $12 \mathrm{~nm}$ thick bow-tie shaped Au bridge (narrowest part $<60 \mathrm{~nm}$ ) is evaporated [Fig. 1(c)] and electrically contacted by two four-terminal thermometers $[5 \mathrm{~nm}$ Ti/65 nm Au, Fig. 1(d)]. The effective temperature drop on a molecule trapped between the two Au contacts depends on the thermal resistances of the Au bridge. Therefore, a short channel length should be used to reduce its thermal resistance which ensures thermalization with the heated $\mathrm{Au}$ contact. On the other hand, very short channels promote direct heating of the "cold" contact by the sample heater. In this study, we chose a short channel length of $1 \mu \mathrm{m}$. Figure 1(e) shows a falsecolor scanning electron microscopy image of a final device. To use these devices for studying the thermoelectric properties of single molecules, we open a nanometer sized gap in the Au bridge by electromigration $^{19}$ followed by self-breaking ${ }^{15}$ to avoid the formation of $\mathrm{Au}$ clusters inside the junction. (a)

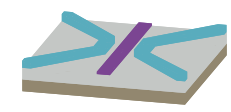

(c)

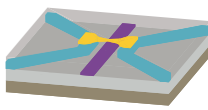

(b)

(d)

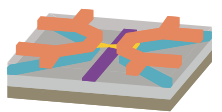

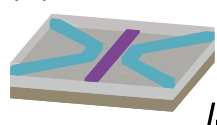

(e)

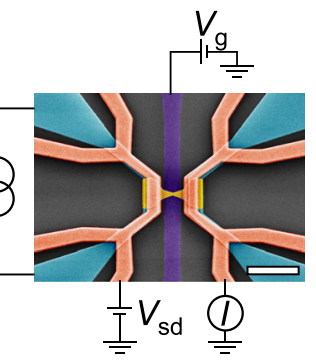

FIG. 1. (a)-(d) Overview of the fabrication process. (a) Fabrication of the local back gate electrode (purple) and sample heaters (blue). (b) Deposition of a thin $\mathrm{Al}_{2} \mathrm{O}_{3}$ insulating layer on top of the whole device. (c) Deposition of a Au bridge (yellow) which is (d) in contact with two four-terminal thermometers (orange). (e) False-color scanning electron micrograph of the single-molecule transistor architecture, consisting of a thin Au bridge (yellow) on top of a gate electrode (purple) connected by two four-terminal thermometers (orange) which are on top of the sample heaters (blue). The schematic circuit diagram indicates the terminals used for $G$ and $I_{\text {th }}$ measurements: a source-drain voltage $V_{\text {sd }}$ can be applied to the drain while a current to ground $I$ is measured at the source. $V_{\mathrm{g}}$ is applied via the back gate with respect to ground. Heater currents $I_{\text {heater }}$ are applied to the sample heater. Scale bar: $2 \mu \mathrm{m}$.
In the following, we describe the methods for estimating $\Delta T$ created by the sample heater after electromigration. We employed two calibration techniques: scanning thermal microscope (SThM) mapping in high vacuum and the resistance thermometer method using the drain and source contacts as thermometers. For the former, we used a home-built high vacuum $\mathrm{SThM}^{20}$ with commercially available (Anasys Instruments, AN-300) doped silicon probes which are geometrically similar to standard micromachined AFM probes. The probe temperature $T_{\text {probe }}$ can be controlled with an integrated heater at the end of the cantilever, which also acts as a calibrated temperature sensor when the tip is in contact with the sample. The electrical response of the probe heater as a function of excess mean probe temperature $\left(\Delta T_{\text {probe }}=T_{\text {probe }}-T_{\text {bath }}\right)$ was calibrated on a heated stage inside the high vacuum chamber, following a procedure described elsewhere. ${ }^{21}$

Two different quantitative SThM methods were employed to estimate $\Delta T$ : the null-point method $^{22}$ and a nonequilibrium thermometry method. ${ }^{23,24}$ In the null-point method, the probe is brought into contact with the sample for different $T_{\text {probe }}$ while the SThM response is recorded. A jump in the SThM response signal is typically observed at the tip-sample mechanical contact when the probe apex and sample are at different temperatures (examples in Fig. S1, supplementary material). The jump is positive/negative when the temperature of the probe apex, $T_{\text {apex }}$, is larger/smaller than that of the sample, $T_{\text {sample }}$, and zero when they are the same. $T_{\text {apex }}$ in contact with the sample with respect to $T_{\text {bath }}$ has been found ${ }^{21}$ to be $88 \%$ of $T_{\text {probe }}$. Using this procedure, we measured the $T_{\text {excess }}=T_{\text {sample }}-T_{\text {bath }}$ of the drain (hot) lead for 4 different powers applied to the sample heater which is plotted in Fig. 2(a). Linear regression yields a conversion factor of $9.8 \pm 1.2 \mathrm{~K} \mathrm{~mW}^{-1}$, with an error originating mainly from the temperature calibration of the probe and the estimation of the jump of the SThM signal (see the supplementary material), especially for low $T_{\text {probe }}$ where the SThM signal noise is comparable to the signal jump.

The second SThM method relies on nonequilibrium thermometry where an AC bias voltage is applied to the sample heater and the resulting variations of $T_{\text {excess }}$ are detected by the SThM tip. The $T_{\text {excess }}$ map is extracted through the relation $T_{\text {excess }}=\Delta T_{\text {probe }} \frac{\Delta V_{\mathrm{AC}}}{\Delta V_{\mathrm{AC}}-\Delta V_{\mathrm{DC}}}$, where $\Delta V_{\mathrm{AC}}$ is the AC SThM response detected at the second harmonic and $\Delta V_{\mathrm{DC}}$ the DC SThM signal due to heat flux from the sample to the tip. Modulation of the sample heater with high frequencies can lead to damping of the SThM signal since thermal equilibrium can only be reached within a time scale $\tau_{\text {th }}$ given by the total thermal capacitance and all thermal resistances of our device. For the temperature mapping, a modulation frequency of $7 \mathrm{~Hz}$ is used, due to limitations in the lowest possible scanning speed, which is slightly larger than $1 / \tau_{\text {th }}$ and which results in a reduction of the SThM signal by about $10 \%$. We account for this damping by rescaling of the $T_{\text {excess }}$ maps using Fig. 4(c). The resulting map for a device with $P_{\text {heat }}=0.38 \mathrm{~mW}$ applied to the sample heater is shown in Fig. 2(b).

From this $T_{\text {excess }}$ map and a line cut through this map in Fig. 2 (c), we observe that for a heating of the hot (left) contact by about $3 \mathrm{~K}$ the cold (right) contact only heats up by about $0.14 \mathrm{~K}$, which yields a very low $\left(T_{\text {cold }}-T_{\text {bath }}\right) / \Delta T \approx 0.05$. This low heating of the cold side allows us to estimate $\Delta T$ from the excess temperature of the drain lead in Fig. 2(a) using $\Delta T \approx T_{\text {excess. }}$ It is worth mentioning that the temperature of the gold bridge differs noticeably from that of the drain and source contacts. This has been observed in previous studies ${ }^{12}$ and would result in an overestimation of $\Delta T$ across the molecule in the 
(a)

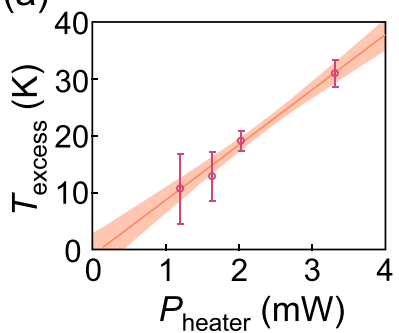

(d)

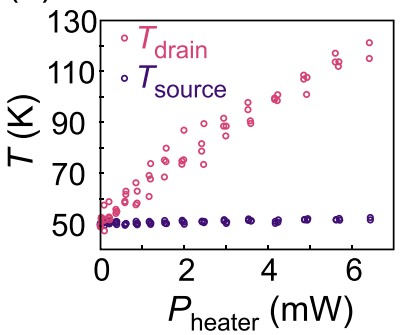

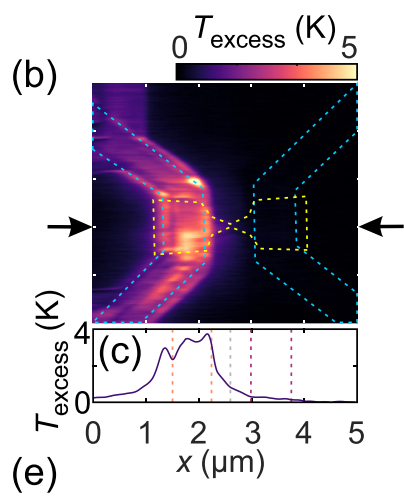

(e)

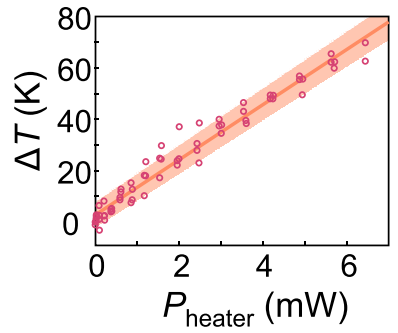

FIG. 2. (a) Results of the SThM null-point method. Excess temperature $T_{\text {excess }}$ of the drain (hot) lead as a function of heater power. The error of the linear fit is indicated by the red shaded area. (b) Temperature map of the device recorded using nonequilibrium thermometry method at $P_{\text {heater }}=0.38 \mathrm{~mW}$. The dotted lines indicate the position of the drain and source leads, and the gold bridge, respectively. A line cut along the device (indicated by arrows) is shown in (c). (d) and (e) Results of the calibration using the resistance thermometer method. (d) Temperature of the drain and source lead as a function of heater power. (e) Temperature drop $\Delta T$ $=T_{\text {drain }}-T_{\text {source }}$ across the junction as a function of heater power. The red shaded area indicates the error of the linear fit.

center of the junction. However, SThM only accesses the phonon (lattice) temperature $T_{\mathrm{ph}}$, and the electron temperature $T_{\mathrm{e}}$ (which drives thermoelectric effects) can be much higher when using an efficient sample heater in direct contact with leads. ${ }^{18}$ Since we cannot access the real drop in $T_{\mathrm{e}}$ on the single-molecule junction, in the remainder of this paper we use the $\Delta T$ between the drain and source lead for calculations, which leads to an underestimation of the thermoelectric coefficients and efficiencies.

The second technique used to estimate $\Delta T$ is the resistance thermometer method. ${ }^{25-27}$ To this end, we use the four contacts connecting the drain and source lead to first measure their 4-terminal resistance as a function of $T_{\text {bath }}$ in a cryostat. Thereafter, the sample temperature is held constant (here $T_{\text {bath }}=50 \mathrm{~K}$ ) and the 4-terminal resistance of the drain and source leads are measured as a function of dissipated heater power. Combining of both measurement results allows estimating $T_{\text {drain }}$ and $T_{\text {source }}$ as a function of heater power $P_{\text {heater }}$ [see Fig. 2(d)]. It can be seen that the (hot) drain lead in direct contact with the sample heater heats up by tens of Kelvin when increasing the heater power while the (cold) source lead stays almost at $T_{\text {bath. }}$. Using these data, we estimate $\Delta T$ as a function of $P_{\text {heater }}$ [Fig. 2(e)]. We find that $\Delta T$ increases linearly with $P_{\text {heater, which }}$ allows to accurately apply small $\Delta T$ biases. Extracting the slope of $10.7 \pm 0.8 \mathrm{~K} \mathrm{~mW}^{-1}$, we find a heating efficiency of $\Delta T /\left(P_{\text {heat }} L\right)$ $=10.7 \pm 0.8 \mathrm{~K} \mathrm{~mW}^{-1} \mu \mathrm{m}^{-1}$ at $50 \mathrm{~K}$. This value is close to the value found using the SThM methods above.

The efficiency found in our devices is orders of magnitude higher than that found in devices with side heaters ${ }^{11}$ and it is comparable to similar devices designed to study thermoelectric properties of nanowires which use a sample heater patterned on top of the leads. ${ }^{18}$ Such a high heating efficiency allows driving systems into the nonlinear regime where $\Delta T$ becomes comparable to, or even exceeds $T_{\text {bath }}$. This is demonstrated in Fig. 2(e) (which was recorded at $T_{\text {bath }}=50 \mathrm{~K}$ ) for $P_{\text {heater }}>5 \mathrm{~mW}$, where $\Delta T>50 \mathrm{~K}$. Moreover, from the data in Fig. 2(d), we find a low $\left(T_{\text {cold }}-T_{\text {bath }}\right) / \Delta T<0.026$, which indicates minimal heating of the cold reservoir and the molecule. This value, which is significantly lower than previously reported values ${ }^{11,18}$ ensures stability of the molecular junction and enables experiments at $T_{\text {bath }}<2 \mathrm{~K}$

In the following, we test the device architecture to measure the thermocurrent of a single $\left[\mathrm{Gd}(\mathrm{tpy}-\mathrm{SH})_{2}(\mathrm{NCS})_{3}\right]$ molecule by immersing the sample in a $0.5 \mathrm{mM}$ molecule solution in dichloromethane after electromigration and self breaking. We observe molecular junction formation indicated by occurrence of gate dependent transport features at $T_{\text {bath }}=1.8 \mathrm{~K}$ in 7 out of 47 junctions. This junction formation yield of $\approx 15 \%$ is similar to values that we typically observe for electromigrated $\mathrm{Au}$ electrodes. ${ }^{28}$ In this paper, we focus on demonstrating the suitability of our junctions for thermoelectric characterization of single molecules and present the data for one selected device.

Figure 3(a) shows the differential conductance $\mathrm{d} I / \mathrm{d} V_{\text {sd }}$ of a molecular junction as a function of bias voltage $V_{\text {sd }}$ and $V_{\mathrm{g}}$. Two regions with low $\mathrm{d} I / \mathrm{d} V_{\text {sd }}$ (yellow) are separated by two crossing lines of high $\mathrm{d} I / \mathrm{d} V_{\text {sd. }}$. These lines are attributed to the borders of the socalled Coulomb diamonds. The current inside the two adjacent diamonds is suppressed due to Coulomb blockade, whereas sequential electron tunneling occurs inside the hour-glass shaped region. ${ }^{3}$

$I_{\text {th }}$ and $G$ were then measured simultaneously in the device configuration shown in Fig. 1(e) following the measurement protocol

(a)
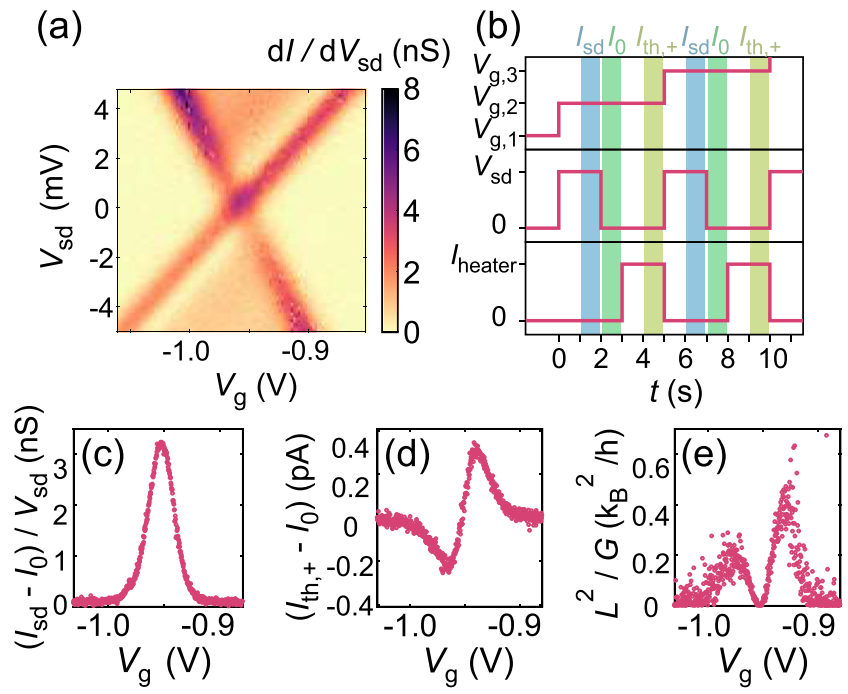

FIG. 3. (a) Differential conductance $d / / d V_{\text {sd }}$ as a function of applied gate $V_{g}$ and bias $V_{\text {sd }}$ voltages. (b) Measurement scheme used to measure $I_{\text {th }}\left(V_{g}\right)$ and $G\left(V_{g}\right)$ as a function of gate voltage. The shaded regions indicate the time windows in which current measurements are performed. (c) Conductance, (d) $I_{\text {th }}$ and (e) power factor as a function of $V_{\mathrm{g}}$. 
depicted in Fig. 3(b). $V_{\mathrm{g}}$ is first ramped to the desired value and a small $V_{\mathrm{sd}}=0.5 \mathrm{mV}$ is applied. After a short settling time $I_{\mathrm{sd}}$ is measured, $V_{\mathrm{sd}}$ is set to zero and an offset current $I_{0}$ may be measured, which can originate from gate leakage currents or offsets in the current preamplifier. Subsequently, a heater current $I_{\text {heater }}=0.1 \mathrm{~mA}(P=2.6 \mu \mathrm{W})$ is applied to the sample heater, followed by a settling time and a measurement of the raw thermocurrent, $I_{\mathrm{th},+}$. These measurement steps are repeated for each gate voltage value. Using the three measured current values, the conductance $G=\left(I_{\text {sd }}-I_{0}\right) / V_{\text {sd }}$ and the thermocurrent $I_{\mathrm{th}}=I_{\mathrm{th},+}-I_{0}$ are calculated. The power factor $S^{2} G$ $=\left(V_{\mathrm{th}} / \Delta T\right)^{2} G=\left(I_{\mathrm{th}} / \Delta T\right)^{2} / G$, which is a measure for the amount of energy that can be generated from a certain $\Delta T$, is thus determined directly.

Figures 3(c) and 3(d) show the results of this measurement on the molecular junction. The conductance $I_{\text {sd }} / V_{\text {sd }}$ peaks at around $V_{\mathrm{g}}=-0.96 \mathrm{~V}$. This indicates the energetic position of the charge degeneracy point where the transition from the $N$ to the $N+1$ charge state of the molecule occurs [corresponds to closing point of the Coulomb diamonds in Fig. 3(a)]. Furthermore, we extract the gate coupling factor $\alpha=C_{\mathrm{g}} /\left(C_{\mathrm{s}}+C_{\mathrm{d}}+C_{\mathrm{g}}\right)=33 \mathrm{meV} \mathrm{V}^{-1}$, from the slopes of the Coulomb diamond following Ref. 29. This gate coupling factor, which is a factor 4-5 higher than the typical values found for devices using $\mathrm{SiO}_{2}$ back gates, ${ }^{30,31}$ enables efficient tuning of the single-molecule junction and allows thermoelectric studies over a wide energy range, of about $\pm 400 \mathrm{meV}$, as estimated using the typical break down voltages of $12-14 \mathrm{~V}$ found in our devices.

Figure 3(d) shows $I_{\text {th }}=I_{\text {th },+}-I_{0}$ as a function of $V_{\mathrm{g}}$, displaying the resulting curve that is S-shaped and changes sign at the charge degeneracy point. This sign change indicates that the transition from electron- to holelike thermocurrents occurs when crossing the charge degeneracy point, in agreement with theoretical predictions. ${ }^{3,32}$ By tuning the system far away from resonance, $I_{\text {th }}$ vanishes. A similar strong gate dependence of the thermoelectric properties has been observed in recent experiments on $\mathrm{C}_{60}{ }^{11,12}$ and $\mathrm{BPDT}^{12}$ molecules. Combining the data in Figs. $3(\mathrm{c})$ and $3(\mathrm{~d})$ and using $\Delta T \approx 30 \mathrm{mK}$ obtained from our calibration allows calculating the gate-dependent power factor $S^{2} G=L^{2} / G$, where $S=-V_{\text {th }} / \Delta T$ is the Seebeck coefficient, $V_{\text {th }}$ is the thermovoltage, and $L=-I_{\text {th }} / \Delta T$ is the thermal response coefficient. The result of this calculation is shown in Fig. 3(e), The power factor can be tuned from zero to about $0.4 k_{\mathrm{B}}^{2} / h$, which is close to the theoretical limit of $(1 / 2.2) k_{\mathrm{B}}^{2} / h$ predicted for a single quantum level. ${ }^{11}$

In the remainder of this paper, we test if the device platform developed in this study is suitable for AC thermoelectric measurements. ${ }^{26}$ For this purpose, an AC current at frequency $f$ is applied to the sample heater and $I_{\text {th }}$ is measured at the second harmonic, $2 f$. As can be shown in Ref. 33, the maximum signal in the second harmonic is at a phase of $90^{\circ}$ with respect to the excitation. Furthermore, the raw data need to be multiplied by a factor of $2 \sqrt{2}$ to convert them from rms to peak-to-peak and to correct the shift in reference when locking to the second harmonic. ${ }^{11}$ Figure 4 (a) shows the AC thermocurrent as a function of gate voltage measured with $f=3 \mathrm{~Hz}$ for the same device discussed above. The line shape and amplitude of the AC measurement match the results of the DC measurement in Fig. 3(d) well. This changes if higher frequencies are used: In Fig. 4(b), the AC thermocurrent measured at fixed gate voltage $\left(V_{\mathrm{g}}=-0.965 \mathrm{~V}\right)$ as a function of modulation frequency of the sample heater is shown. (a)
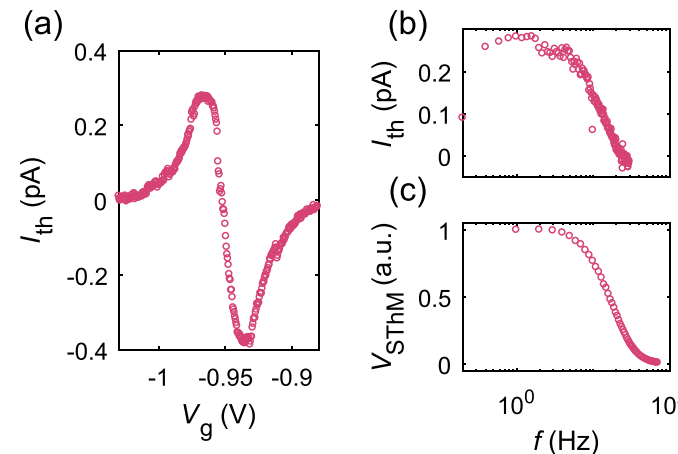

(c)

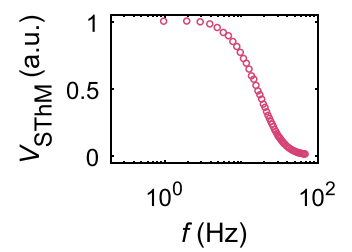

FIG. 4. (a) AC thermocurrent $\left(f=3 \mathrm{~Hz}, I_{\text {heater }}=0.1 \mathrm{~mA}, P_{\text {heater }}=26 \mu \mathrm{W}\right)$ as a function of gate voltage. (b) Thermocurrent at $V_{g}=-0.965 \mathrm{~V}$ as a function of modulation frequency of the sample heater. (c) SThM signal on the drain (hot) contact as a function of modulation frequency of the sample heater.

Above a frequency of about $3 \mathrm{~Hz}$ the signal amplitude drops from its DC value to zero when reaching frequencies of about $30 \mathrm{~Hz}$. This can be explained by the thermal equilibrium time of the system as discussed above. To illustrate this, the SThM signal measured on the hot contact as a function of sample heater excitation frequency is shown in Fig. 4(c). A similar trend to that for the thermocurrent signal can be observed where a deviation from the DC signal strength occurs at $f>3 \mathrm{~Hz}$.

In summary, we developed a device architecture and a robust measurement protocol that allows measuring the thermoelectric properties of single molecules at cryogenic temperatures, over a wide energy range. The close proximity of the sample heater to the electrical contacts yields a high heating efficiency and low global heating of the molecular junction itself. This ensures device stability and allows the accurate study of thermoelectric effects over wide $\Delta T$ ranges. Furthermore, we demonstrate that the gate dependent thermocurrent and conductance can be measured in parallel and that the devices are suitable for AC measurements, if the excitation frequency is chosen to be smaller than the thermal response time of the system. The devices presented in this study could thus be readily used to study the thermoelectric properties of single molecules in the nonlinear regime ${ }^{34}$ or to investigate the thermoelectric response of single-molecule magnets ${ }^{9}$ or high-spin molecules in the Kondo regime. ${ }^{10}$ What is more, the Gdbased molecules used in this study are promising candidates for observing single-molecule magnetocooling effects ${ }^{35}$ which are now within experimental reach.

See the supplementary material for individual approach curves and calibration details of the null-point method, AC thermovoltage measurements with an estimation of the Seebeck coefficient of the junction, and details on the estimation of $\Delta T$ from the SThM data.

This work was supported by the EC H2020 FET Open project 767187 QuIET and ERC (Nos. StG-OptoQMol-338258 and CoGMMGNRs-773048). P.G. and J.R. acknowledge Marie SkodowskaCurie Individual Fellowships (Grant Nos. TherSpinMol-748642 and SpinReMag-707252) from the European Unions Horizon 2020 research and innovation programme. 


\section{REFERENCES}

${ }^{1}$ H. Valkenier, C. M. Gudon, T. Markussen, K. S. Thygesen, S. J. van der Molen, and J. C. Hummelen, "Cross-conjugation and quantum interference: A general correlation?” Phys. Chem. Chem. Phys. 16, 653-662 (2014).

${ }^{2}$ C. M. Finch, V. M. García-Suárez, and C. J. Lambert, "Giant thermopower and figure of merit in single-molecule devices,” Phys. Rev. B 79, 033405 (2009).

${ }^{3}$ P. Gehring, J. M. Thijssen, and H. S. J. van der Zant, "Single-molecule quantum-transport phenomena in break junctions," Nat. Rev. Phys. 1, 381-396 (2019).

${ }^{4} \mathrm{~J}$. Koch, F. von Oppen, and A. V. Andreev, "Theory of the franck-condon blockade regime," Phys. Rev. B 74, 205438 (2006).

${ }^{5}$ W. Liang, M. P. Shores, M. Bockrath, J. R. Long, and H. Park, "Kondo resonance in a single-molecule transistor," Nature 417, 725-729 (2002).

${ }^{6} \mathrm{~J}$. de Bruijckere, P. Gehring, M. Palacios-Corella, M. Clemente-León, E. Coronado, J. Paaske, P. Hedegård, and H. S. J. van der Zant, "Ground-state spin blockade in a single-molecule junction," Phys. Rev. Lett. 122, 197701 (2019).

${ }^{7}$ J. Koch, F. von Oppen, Y. Oreg, and E. Sela, "Thermopower of single-molecule devices," Phys. Rev. B 70, 195107 (2004).

${ }^{8}$ J. K. Sowa, J. A. Mol, and E. M. Gauger, "Marcus theory of thermoelectricity in molecular junctions,” J. Phys. Chem. C 123, 4103-4108 (2019).

${ }^{9}$ R.-Q. Wang, L. Sheng, R. Shen, B. Wang, and D. Y. Xing, "Thermoelectric effect in single-molecule-magnet junctions," Phys. Rev. Lett. 105, 057202 (2010).

${ }^{10}$ T. A. Costi and V. Zlatić, "Thermoelectric transport through strongly correlated quantum dots," Phys. Rev. B 81, 235127 (2010).

${ }^{11}$ P. Gehring, A. Harzheim, J. Spice, Y. Sheng, G. Rogers, C. Evangeli, A. Mishra, B. J. Robinson, K. Porfyrakis, J. H. Warner, O. V. Kolosov, G. A. D. Briggs, and J. A. Mol, "Field-effect control of graphenefullerene thermoelectric nanodevices,” Nano Lett. 17, 7055-7061 (2017).

${ }^{12}$ Y. Kim, W. Jeong, K. Kim, W. Lee, and P. Reddy, "Electrostatic control of thermoelectricity in molecular junctions," Nat. Nanotechnol. 9, 881-885 (2014).

${ }^{13}$ J. Moon, J.-H. Kim, Z. C. Chen, J. Xiang, and R. Chen, "Gate-modulated thermoelectric power factor of hole gas in gesi coreshell nanowires," Nano Lett. 13, 1196-1202 (2013).

${ }^{14}$ T. A. Su, M. Neupane, M. L. Steigerwald, L. Venkataraman, and C. Nuckolls, "Chemical principles of single-molecule electronics," Nat. Rev. Mater. 1, 16002 (2016).

${ }^{15} \mathrm{~K}$. ONeill, E. A. Osorio, and H. S. J. van der Zant, "Self-breaking in planar fewatom Au constrictions for nanometer-spaced electrodes," Appl. Phys. Lett. 90, 133109 (2007).

${ }^{16}$ P. Gehring, H. Sadeghi, S. Sangtarash, C. S. Lau, J. Liu, A. Ardavan, J. H. Warner, C. J. Lambert, G. A. D. Briggs, and J. A. Mol, "Quantum interference in graphene nanoconstrictions," Nano Lett. 16, 4210-4216 (2016).

${ }^{17}$ S. Nazarpour, A. Cirera, and M. Varela, "Material properties of aupd thin alloy films," Thin Solid Films 518, 5715-5719 (2010).

${ }^{18}$ J. G. Gluschke, S. Fahlvik Svensson, C. Thelander, and H. Linke, Nanotechnology 25, 385704 (2014).
${ }^{19}$ H. Park, A. K. L. Lim, A. P. Alivisatos, J. Park, and P. L. McEuen, "Fabrication of metallic electrodes with nanometer separation by electromigration," Appl. Phys. Lett. 75, 301-303 (1999).

${ }^{20}$ M. E. Pumarol, M. C. Rosamond, P. Tovee, M. C. Petty, D. A. Zeze, V. Falko, and O. V. Kolosov, "Direct nanoscale imaging of ballistic and diffusive thermal transport in graphene nanostructures," Nano Lett. 12, 2906-2911 (2012).

${ }^{21}$ P. Tovee, M. Pumarol, D. Zeze, K. Kjoller, and O. Kolosov, "Nanoscale spatial resolution probes for scanning thermal microscopy of solid state materials," J. Appl. Phys. 112, 114317 (2012).

${ }^{22}$ G. Hwang, J. Chung, and O. Kwon, "Enabling low-noise null-point scanning thermal microscopy by the optimization of scanning thermal microscope probe through a rigorous theory of quantitative measurement," Rev. Sci. Instrum. 85, 114901 (2014).

${ }^{23}$ F. Menges, P. Mensch, H. Schmid, H. Riel, A. Stemmer, and B. Gotsmann, "Temperature mapping of operating nanoscale devices by scanning probe thermometry," Nat. Commun. 7, 10874 (2016).

${ }^{24}$ A. Harzheim, J. Spiece, C. Evangeli, E. McCann, V. Falko, Y. Sheng, J. H. Warner, G. A. D. Briggs, J. A. Mol, P. Gehring, and O. V. Kolosov, "Geometrically enhanced thermoelectric effects in graphene nanoconstrictions," Nano Lett. 18, 7719-7725 (2018).

${ }^{25}$ P. Kim, L. Shi, A. Majumdar, and P. L. McEuen, "Thermal transport measurements of individual multiwalled nanotubes," Phys. Rev. Lett. 87, 215502 (2001).

${ }^{26}$ J. P. Small, L. Shi, and P. Kim, "Mesoscopic thermal and thermoelectric measurements of individual carbon nanotubes," Solid State Commun. 127, 181-186 (2003).

${ }^{27}$ J. P. Small, K. M. Perez, and P. Kim, "Modulation of thermoelectric power of individual carbon nanotubes,” Phys. Rev. Lett. 91, 256801 (2003).

${ }^{28}$ E. Burzurí, R. Gaudenzi, and H. S. J. van der Zant, "Observing magnetic anisotropy in electronic transport through individual single-molecule magnets," J. Phys.: Condens. Matter 27, 113202 (2015).

${ }^{29}$ R. Hanson, L. P. Kouwenhoven, J. R. Petta, S. Tarucha, and L. M. K. Vandersypen, "Spins in few-electron quantum dots," Rev. Mod. Phys. 79, 1217-1265 (2007).

${ }^{30}$ E. A. Osorio, T. Bjørnholm, J.-M. Lehn, M. Ruben, and H. S. J. van der Zant, "Single-molecule transport in three-terminal devices," J. Phys.: Condens. Matter 20, 374121 (2008).

${ }^{31}$ P. Gehring, J. K. Sowa, J. Cremers, Q. Wu, H. Sadeghi, Y. Sheng, J. H. Warner, C. J. Lambert, G. A. D. Briggs, and J. A. Mol, "Distinguishing lead and molecule states in graphene-based single-electron transistors," ACS Nano 11, 5325-5331 (2017).

${ }^{32}$ L. Rincón-García, C. Evangeli, G. Rubio-Bollinger, and N. Agraït, "Thermopower measurements in molecular junctions," Chem. Soc. Rev. 45, 4285-4306 (2016).

${ }^{33}$ Y. M. Zuev, "Nanoscale thermoelectric energy conversion," $\mathrm{PhD}$ thesis (Columbia University, 2011).

${ }^{34}$ D. Sánchez and R. López, "Scattering theory of nonlinear thermoelectric transport," Phys. Rev. Lett. 110, 026804 (2013).

${ }^{35} \mathrm{G}$. Karotsis, M. Evangelisti, S. Dalgarno, and E. Brechin, "A calix[4] arene 3d/4f magnetic cooler," Angew. Chem. Int. Ed. 48, 9928-9931 (2009). 